\title{
Spatial distribution and functional feeding groups of aquatic insect communities in Serra da Bocaina streams, southeastern Brazil
}

\author{
Distribuição espacial e categorização funcional trófica de comunidades \\ de insetos aquáticos em rios na Serra da Bocaina, Sudeste Brasil
}

Ana Lucia Henriques de Oliveira and Jorge Luiz Nessimian

Laboratório de Entomologia, Departamento de Zoologia, Instituto de Biologia,

Universidade Federal do Rio de Janeiro - UFRJ, Bloco - A, sala 107, CCS,

Ilha do fundão, Cidade Universitária, CP 68044, CEP 21941-971, Rio de Janeiro, RJ, Brazil e-mail: anahenri@biologia.ufrj.br; jnessimian@gmail.com

\begin{abstract}
Aim: The aim of this paper was to study the spatial distribution and functional feeding structure of aquatic insect communities of 18 streams at different altitudes in Mambucaba River Basin, Serra da Bocaina, Southeastern Brazil; Methods: Samples were collected in two consecutive dry periods (August of 2003 and 2004) being sampled four substrate types in each stream: litter from riffles, litter from pools, rocks and gravel; Results: We identified 75,581 aquatic insect individuals belonging to 201 taxa. Most of the fauna was found in litter substrates (64\%), and riffle litter substrate had the majority of the specimens (32,572 individuals). Gravel was the substrate with highest values of richness (29.84 taxa expected for 187 individuals; rarefaction method) and Shannon's diversity $\left(H^{\prime}=2.370\right)$. Rock substrate showed the lowest richness (20.24 taxa). Distribution of taxa across substrates shows that only 28 taxa are restricted to a single substrate, while 78 taxa occurred in all substrates. The indicator analysis showed that 20 taxa were characteristic of pool litter, 25 of riffle litter, 22 of gravel and only nine to rock. In relation to organic and inorganic substrates, 29 taxa were characteristic of litter, and 17 of inorganic substrates. Cluster analysis based on Bray-Curtis dissimilarity index and UPGMA linkage method showed that aquatic insects were distributed according to substrate and food resource. In all substrates, the main functional feeding group was collector-gatherer $(40.64 \%)$, and the least representative was shredder (6.67\%). ANOVA and Tukey HSD tests showed that collector-gatherers and shredders were predominant in pool substrates, collector-filters in riffle substrate and scrapers in hard substrates; Conclusion: Ours results show that independently of stream or altitude, substrate of the same type have similar faunal assemblages. The abundance and relative proportion of the functional feeding group showed variation across habitats.
\end{abstract}

Keywords: distribution, habitats, substrates, benthic macroinvertebrates.

Resumo: Objetivo: $\mathrm{O}$ objetivo deste artigo foi estudar a distribuição espacial e a categorização funcional trófica da comunidade de insetos aquáticos de 18 riachos em diferentes altitudes na Bacia do Rio Mambucaba, Serra da Bocaina, Sudeste do Brasil; Métodos: As amostras foram coletadas em dois períodos secos consecutivos (agosto de 2003 e 2004), sendo coletados em quatro tipos de substratos: folhiço em correnteza, folhiço de fundo, pedras e cascalho; Resultados: Foram identificados 75.581 indivíduos de insetos aquáticos pertencentes a 201 táxons. Os substratos com folhiço concentraram a maior parte da fauna (64\%), sendo o folhiço em correnteza o substrato com maior abundância de indivíduos (32.572). O cascalho apresentou os maiores valores de riqueza (29,84 táxons esperado em 187 indivíduos; método da rarefação) e diversidade de Shannon $\left(H^{\prime}=2,370\right)$. A menor riqueza $(20,24$ táxons) foi observada no substrato pedra. A distribuição dos táxons entre os substratos mostrou 28 táxons restritos a um tipo de substrato, enquanto 78 táxons ocorreram em todos os substratos. Vinte táxons foram indicativos para folhiço de fundo, 26 para folhiço em correnteza, 22 para cascalho e nove táxons para pedra. Em relação aos substratos orgânicos e inorgânicos, 29 táxons foram característicos de folhiço, e 17 de substratos inorgânicos. A análise de classificação baseada no índice de Bray-Curtis e método de ligação UPGMA indicou que os insetos aquáticos se distribuíram em relação ao tipo de substrato e disponibilidade de alimento. Em todos os substratos, a principal categoria funcional foi coletor-catador $(40,64 \%)$, enquanto fragmentador $(6,67 \%)$ a menor abundância. ANOVA e Teste HSD de Tukey mostraram que os coletor-catadores e fragmentador foram predominantes nos substratos de folhiço em depósitos, coletores-filtradores nos substrates de correnteza e raspadores nos substratos duros; Conclusóes: Nossos resultados mostraram que independentemente do rio ou altitude, os substratos de um mesmo tipo apresentam assembléias similares. A abundância e a proporção relativa dos grupos funcionais tróficos mostrou variação entre os habitats.

Palavras-chave: distribuiçấo, habitat, substratos, macroinvertebrados bentônicos. 


\section{Introduction}

Habitat preferences of benthic macroinvertebrates result from the balance of a variety of requirements of organisms (Beisel et al., 1998). According to Pardo and Armitage (1997), the nature of the substratum together with flow patterns result in a patchy distribution of mesohabitats, each inhabited by a particular assemblage of species. The habitats for aquatic insect communities in riverine ecosystems can be interpreted within the framework of various spatio-temporal scales (Subramanian and Sivaramakrishnan, 2005). The spatial distribution of some aquatic insects seems to respond to some environmental parameters of major influence, such as current, temperature, oxygen concentration, $\mathrm{pH}$, substrate particle size and food supply (Cummins and Lauff, 1969).

Lotic ecosystems have a continuous and unidirectional water movement which is responsible for many processes, like organic matter transport, sediment deposition and longitudinal gradients inside the drainage basin (Vannote el al., 1980; Williams and Feltmate, 1994). The water flow influences the substrate particle size and the amount of available food, promoting release and/ or remotion of nutrients. Flow velocity is regarded as the main factor determining distribution of organisms in lotic systems (Minshall, 1984; Ward, 1992). The spatial availability of substrate, at local scale, is represented by alternation between pools and riffles. Temporally, this variability is produced by variation of flow velocity, which can remove, mix and redeposit substrates (Hynes, 1970; Minshall, 1984).

In aquatic habitats, the substrate is often a composite of various materials and particle sizes arranged in mosaics (Ward, 1992). The organic substrate provides protection and habitat space, has fundamental importance as food source, and in many instances may constitute a limiting factor. Allochthonous organic matter, mainly leaves from riparian vegetation, is a major energy source for woodland streams or streams with well developed riparian corridors of vegetation (Benfield, 1997). According to Benfield (1997), the litterfall may include leaves and leaf fragments, floral parts, bark, wood (branches and twigs), and fruits, which may reach streams by direct fall or lateral movement. Litterfall contribution is predicted to be highest in headwater streams and gradually diminishes at high order streams (Vannote et al., 1980).

Cummins (1973) classified organic matter in lotic systems by size - the coarse particulate organic matter (CPOM, > $1 \mathrm{~mm}$ ) being composed by leaves and wood from riparian vegetation and macrophytes; fine particulate organic matter (FPOM, $0.5 \mu \mathrm{m}$ $<1 \mathrm{~mm}$ ) originated from a great variety of resources, including CPOM fragmentation, periphyton, algae, and microorganisms; and dissolved organic matter $(\mathrm{DOM},<0.5 \mu \mathrm{m})$, found in suspension in the water column. The availability of food is an obvious factor controlling the occurrence and abundance of species. Generally, species occur, or are common, only where their food is readily available, although few runningwater invertebrates are very specialized in their diets (Hynes, 1970). In relation to functional feeding groups, invertebrates can be classified as: collectors (gatherers or filterers), shredders, scrapers, and predators (Cummins and Klug, 1979; Merritt and Cummins, 1996). Collectors feed on fine particulate organic matter (FPOM), and can be classified in collector-filter, feeding on suspension particles of FPOM or collector-gatherer, acquiring FPOM from interstices in the bottom sediments. Shredders are macroinvertebrates whose mouthparts allow them to consume leaf litter effectively and, therefore, perform an important role in the transformation of CPOM material to FPOM in lotic systems, which in turn can used as food by collector-gatherers and filter-feeders. Scrapers harvest attached algae from substrate surface and predators are defined as those invertebrates that capture and consume live prey (Cummins and Klug, 1979; Wallace and Webster, 1996; Graça et al., 2001; Merritt et al., 2005).

The number of ecological studies on aquatic macroinvertebrates in Atlantic Forest streams in southeastern Brazil have been increasing considerably in the last decade (e.g. Baptista et al., 2001a,b, 2007; Callisto et al., 2001; Roque and Trivinho-Strixino, 2001; Moulton and Magalhães, 2003; Silveira et al., 2005, 2006; Souza and Moulton, 2005). The knowledge on the spatial distribution and functional feeding groups of aquatic insects constitute an important tool in biomonitoring programs. Besides, it provides a basic knowledge for the identification of policies and proposal for conservation and maintenance use of natural resources of a given area.

The aim of this study was to describe the composition and spatial distribution of aquatic insects communities of 18 streams at different altitudes in Mambucaba River Basin at Serra da Bocaina, Southeastern Brazil, and analyze the functional feeding trophic structure of the community in different habitats. 


\section{Material and Methods}

\subsection{Study area}

The study was conducted in 18 tributaries of Mambucaba River Basin in different altitudes at Serra da Bocaina National Park (220 40' S and $23^{\circ} 25^{\prime} \mathrm{S}, 4^{\circ} 20^{\prime} \mathrm{W}$ and $45^{\circ} 00^{\prime} \mathrm{W}$ ) in the municipalities of Angra dos Reis and Paraty (Rio de Janeiro State) and São José do Barreiro (São Paulo State) (Figure 1). The vegetation at all sampling sites is composed by primary or secondary Tropical Atlantic Rain Forest. All streams have forested catchments, clear water, riverbed with rocks, gravel, cobbles and sand, and are free of point pollution. Mosses and algae biofilm cover rocky substrates in rapid reaches, while leaf/wood accumulated in pool areas.

\subsection{Sampling}

Samples were collected in two consecutive dry periods (August of 2003 and 2004). Four substrate types were sampled in each stream: litter from riffles (LR), litter from pools (LP), rocks from riffles (RO) and gravel (GR) from areas with moderate current. Three samples of each substrate were collected using a Surber net (area of $900 \mathrm{~cm}^{2}$ and mesh of $185 \mu \mathrm{m})$. The following variables were measured in situ: electric conductivity $\left(\mu \mathrm{S} . \mathrm{cm}^{-1}\right), \mathrm{pH}$, depth $(\mathrm{m})$, width $(\mathrm{m})$, water current velocity $(\mathrm{m} / \mathrm{s})$, and flow $\left(\mathrm{m}^{3} / \mathrm{s}\right)$ (Table 1). Samples were fixed and conserved

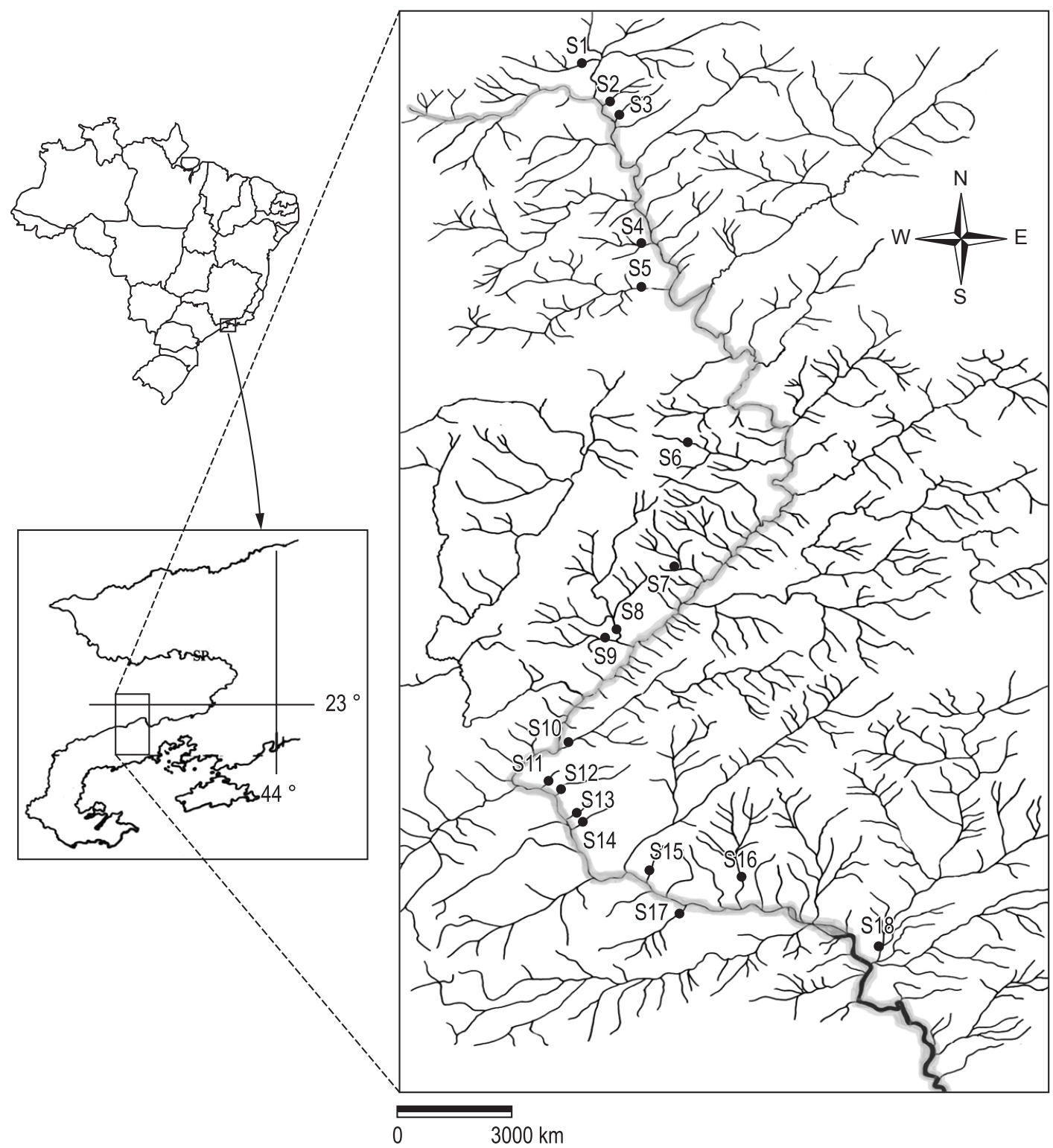

Figure 1. Map of Mambucaba River basin, showing the eighteen $(\bullet)$ sampling sites. Mambucaba River is marked gray. 


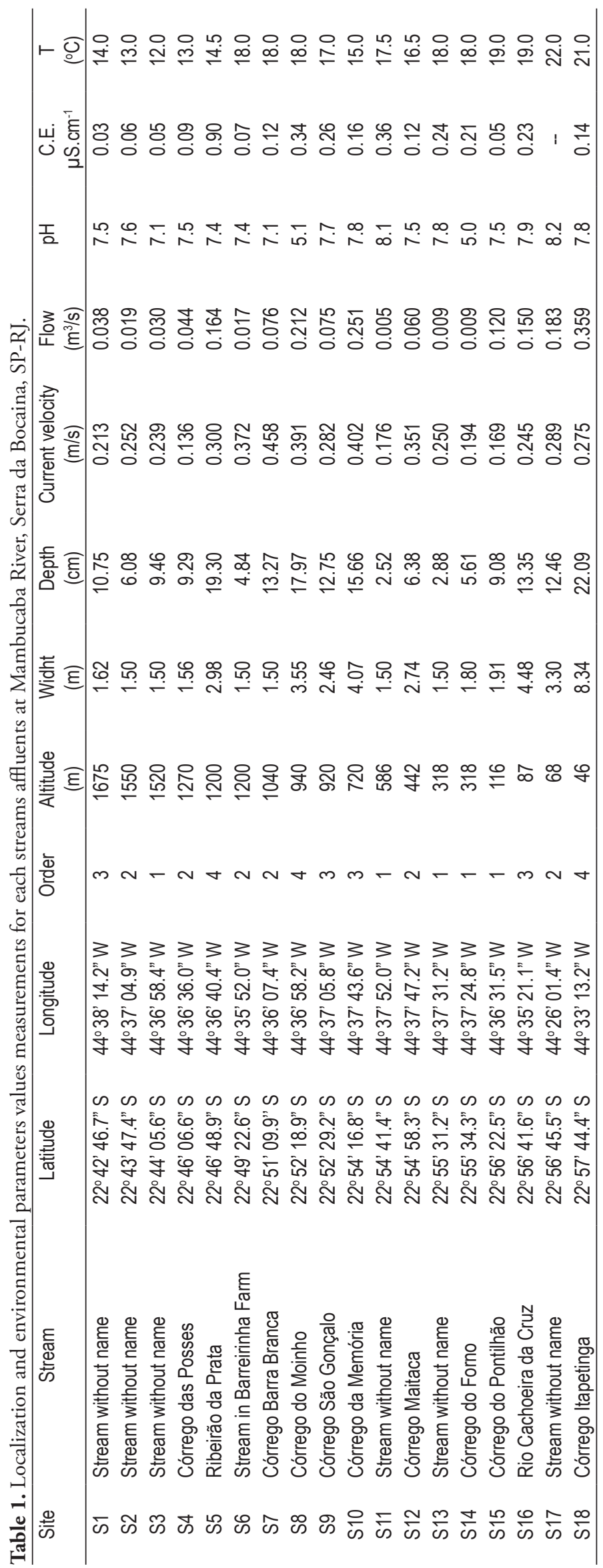


in $80 \%$ alcohol and sorted under stereoscopic microscope. A single sample from each substrate was composed by pooling three Surber sample units from the same substrate per stream.

Identification of organisms was performed to the lower taxonomic level possible, except for Diptera (only identified at family level), with aid of keys and taxonomic descriptions (Carvalho, 1989; Belle, 1992; Angrisano, 1995; Merritt and Cummins, 1996; Nieser and Melo, 1997; Carvalho and Calil, 2000; Carvalho et. al., 2002; Da-Silva et al., 2003; Olifiers et al., 2004; Salles et al., 2004; Pes et al., 2005; Dias et al., 2006) and with the aid taxonomic specialists. Functional feeding groups of aquatic insects were based on Merritt and Cummins (1996), Merritt et al. (2005) and Baptista et al. (2006).

\subsection{Data analysis}

Species diversity was calculated using the Shannon-Wiener index (Elliott, 1977; Ludwig and Reynolds, 1988), and the taxonomic richness values in the different substrates were compared using a rarefaction method. The rarefied taxa richness expected was calculated to occur in 187 individuals, the total abundance obtained in the smallest sample. The species Indicator Value analysis (IV) of Dufrêne and Legendre (1997) was used to identify taxa or functional feeding group characteristic of each substrate or groups of substrates (organic or inorganic). The significance of the statistics was assessed using Monte Carlo tests (McCune and Mefford, 1999). We used classification analysis to assess similarities among substrate samples. Groups were obtained using the Bray-Curtis dissimilarity index and the UPGMA linkage method. The analysis was obtained using the program NTSYS version 1.70 (Rohlf, 1992). In order to assess whether functional feeding group abundances were different among substrates, an Analysis of Variance (ANOVA) was performed, followed by Tukey HSD test (Statsoft, 2001).

\section{Results}

\subsection{Environmental features}

Water temperature values varied according to the altitude (Table 1). The streams located above $1250 \mathrm{~m}$ a.s.l. present temperatures lower than $14.5^{\circ} \mathrm{C}$, while those located below $200 \mathrm{~m}$ present temperatures higher than $19.0{ }^{\circ} \mathrm{C}$. The streams S14 (located at $318 \mathrm{~m}$ a.s.l) and S8 (located at $940 \mathrm{~m}$ a.s.l) showed the lowest $\mathrm{pH}$ values 5.0 and 5.1 respectively, and the highest $\mathrm{pH}$ values were measured at streams
S11 (8.1) (located at $586 \mathrm{~m}$ a.s.l) and S17 (8.2) (located at $68 \mathrm{~m}$ a.s.l). Electric conductivity values were higher at site S5 $\left(0.90 \mu \mathrm{S} . \mathrm{cm}^{-1}\right)$, located at $1.200 \mathrm{~m}$ a.s.l, and the lower values were measured at $S 1\left(0.03 \mu \mathrm{S} . \mathrm{cm}^{-1}\right)$, located at $1675 \mathrm{~m}$ a.s.l.

\subsection{Spatial distribution and faunal composition}

A total of 75,581 aquatic insect individuals were identified, belonging to 201 taxa, distributed among the orders Ephemeroptera, Odonata, Plecoptera, Blattodea, Hemiptera, Megaloptera, Coleoptera, Trichoptera, Lepidoptera, and Diptera (Table 2). About $64 \%$ of the aquatic insect fauna occurred in organic substrates, including both riffle and pool litter (32,572 and 17,752 individuals respectively.). Riffle litter presented the highest abundance, corresponding to $41.92 \%$ of the total. Paragripopteryx sp. (6.32\%), Smicridea sp.1 (4.16\%), Nectopsyche sp.1 (4.19\%), Grumicha sp.2 (3.34\%), and Anacroneuria sp. (3.30\%) were the most abundant taxa in this substrate. Besides that, some caddisfly taxa were observed preferentially in this substrate, such as Atopsyche sp., Chimarra sp., Contulma sp., and Phylloicus sp.2. The highest richness (29.84 taxa expected to occur with 187 individuals) and Shannon's diversity $\left(\mathrm{H}^{\prime}=2.370\right)$ were found in gravel substrate, which also showed the lowest amount of individuals (10,640 ind.). Rock substrate showed the lowest richness (20.24 taxa) and presented 14,617 individuals (Figure 2). In this substrate, scrapers like Grumichella sp. (12.58\%), Baetodes sp. (6.34\%) and Hydroptilidae taxa presented their highest abundances.

Distribution of taxa across substrates shows that only 28 taxa were restricted to a single substrate, while 78 taxa occurred in all substrates. In fast flowing areas, Camelobaetidius sp., Leucotrichia sp., Metrichia sp. 5 and Rhyacopsyche sp. 2 were found exclusively in rock. Guaranyperla sp., Suphisellus sp., Enocchrus sp., Oocyclus sp., Hydrophilidae sp. 1 and Ochrotrichia sp. only occurred in riffle litter. Cyanogomphus sp., Epigomphus sp., Limnocoris intermedius, Berosus sp., Phylloicus sp.4, Austrotinodes sp. and Macronema sp. occurred only in pool litter. Askola sp.2, Macrothemis sp., Coleopterocoris hungerford, Plactynectes sp., Stegoelmis sp., Marilia sp.2, Marilia sp.5, Polycentropodidae sp. 1 and Sericostomatidae sp. 1 were found exclusively in gravel. The results of Indicator Value analysis performed by each substrate or groups of substrates are in Table 3. Riffle litter was the substrate with highest number of characteristics taxa (25) and rock substrate had only nine indicator 
Table 2. Taxa and functional feeding groups (FFG) found to each substrates at Serra da Bocaina streams.

\begin{tabular}{|c|c|c|c|c|c|c|c|}
\hline & & Taxa & FFG & $\mathrm{LP}$ & LR & GR & RO \\
\hline \multirow[t]{33}{*}{ Ephemeroptera } & Baetidae & Americabaetis & CG & 7 & 91 & 10 & 21 \\
\hline & & Baetodes & SC & 9 & 58 & 76 & 747 \\
\hline & & Camelobaetidius & SC & - & - & - & 39 \\
\hline & & Cloeodes & CG & 12 & 2 & 11 & 30 \\
\hline & & Paracloeodes & SC & 14 & 4 & 1 & - \\
\hline & & $\begin{array}{l}\text { Zelusia principalis Lugo-Ortiz \& } \\
\text { McCafferty, } 1995\end{array}$ & CG & 69 & 9 & 2 & - \\
\hline & Caenidae & Caenis & CG/SC & 120 & 1 & 2 & 1 \\
\hline & Euthyplociidae & Campylocia & $\mathrm{CF} / \mathrm{SH}$ & 4 & - & 29 & - \\
\hline & Leptohyphidae & Leptohyphes & CG & 33 & 76 & 53 & 5 \\
\hline & & Leptohyphodes & CG & 511 & 7 & 122 & 2 \\
\hline & & Traverhyphes & CG & 52 & 36 & 110 & 4 \\
\hline & & Tricorytopsis & CG & 18 & 4 & 144 & 49 \\
\hline & & Tricorythodes & CG & 13 & 7 & 40 & 2 \\
\hline & Leptophlebiidae & Askola froehlichi Peters, 1969 & CG/SC & 50 & - & 47 & - \\
\hline & & Askola sp.1 & CG/SC & 160 & - & 6 & - \\
\hline & & Askola sp.2 & CG/SC & 48 & - & - & - \\
\hline & & $\begin{array}{l}\text { Farrodes carioca Domínguez, Molineri \& } \\
\text { Peters, } 1996\end{array}$ & CG/SC & 257 & 101 & 344 & 10 \\
\hline & & Hagenulopsis diptera Ulmer, 1920 & CG/SC & 137 & 88 & 299 & 14 \\
\hline & & Homothraulus & CG/SC & 2 & 4 & 3 & - \\
\hline & & $\begin{array}{l}\text { Hylister plaumanni Domínguez \& } \\
\text { Flowers, } 1989\end{array}$ & CG/SC & - & 25 & 2 & 4 \\
\hline & & Massartela alegrettae Ulmer, 1945 & CG/SC & 11 & - & 1 & - \\
\hline & & Massartela brieni Lestage, 1920 & CG/SC & 13 & - & 2 & - \\
\hline & & Massartela sp.1 & CG/SC & 5 & 3 & - & - \\
\hline & & Massartela sp.2 & CG/SC & 1 & 3 & - & - \\
\hline & & Miroculis froehlichi Savage \& Peters, 1983 & CG/SC & 630 & 18 & 81 & 3 \\
\hline & & Needhamella & CG/SC & 15 & 4 & - & 3 \\
\hline & & aff. Perissophlebiodes & CG/SC & 2 & - & 1 & - \\
\hline & & $\begin{array}{l}\text { Thraulodes itatiajanus Traver \& } \\
\text { Edmunds, } 1967\end{array}$ & CG/SC & 6 & 46 & 271 & 24 \\
\hline & & Thraulodes sp.1 & CG/SC & - & 1 & 21 & 1 \\
\hline & & aff. Thraulodes & CG/SC & 11 & 5 & 52 & 1 \\
\hline & & Ulmeritoides sp.1 & CG & 238 & - & 2 & - \\
\hline & & Ulmeritoides sp.2 & SC & 42 & 1 & 5 & - \\
\hline & Melanemerellidae & Melanemerella brasiliana Ulmer, 1920 & SH & 45 & 17 & 5 & - \\
\hline \multirow[t]{12}{*}{ Odonata } & Aeshnidae & Limnetron debile Karsch, 1891 & $P$ & 11 & - & 1 & - \\
\hline & & Limnetron sp.1 & $P$ & 7 & 2 & - & - \\
\hline & Calopterygidae & Hetaerina & $P$ & 42 & 25 & 15 & 3 \\
\hline & Coenagrionidae & Argia & $P$ & 2 & - & 14 & - \\
\hline & Corduliidae & Neocordulia & $P$ & 25 & - & 3 & - \\
\hline & Gomphidae & Cyanogomphus & $P$ & 1 & - & - & - \\
\hline & & Epigomphus & $P$ & 1 & - & - & - \\
\hline & & Progomphus gracilis Hagen in Selys, 1854 & $P$ & 13 & 1 & 11 & - \\
\hline & & Progomphus sp. 1 & $P$ & 2 & 1 & 9 & - \\
\hline & Libellulidae & Brechmorrhoga & $P$ & 2 & 3 & 18 & - \\
\hline & & Macrothemis & $P$ & - & - & 1 & - \\
\hline & Megapodagrionidae & Heteragrion & $P$ & 44 & 7 & 11 & - \\
\hline Blattodea & Blattidae & & CG & 1 & 20 & 3 & - \\
\hline
\end{tabular}

$\mathrm{LP}=$ litter from pool, $\mathrm{LR}=$ litter from riffle, $\mathrm{GR}=$ gravel and $\mathrm{RO}=$ rocks. $\mathrm{CG}=$ collector-gatherers, $\mathrm{CF}=$ collectorfilterers, $\mathrm{SC}=$ scrapers, $\mathrm{SH}=$ shredders, $\mathrm{P}=$ predators. 
Table 2. Continued...

\begin{tabular}{|c|c|c|c|c|c|c|c|}
\hline & & Taxa & FFG & LP & LR & GR & RO \\
\hline \multirow[t]{7}{*}{ Plecoptera } & \multirow[t]{4}{*}{ Gripopterigydae } & Gripopteryx & CG/SC & 4 & 67 & 20 & 191 \\
\hline & & Guaranyperla & CG & - & 3 & - & - \\
\hline & & Paragripopteryx & CG/SH & 297 & 1504 & 361 & 329 \\
\hline & & Tupiperla & CG & 184 & 230 & 57 & 10 \\
\hline & \multirow[t]{3}{*}{ Perlidae } & Anacroneuria & P & 40 & 811 & 177 & 71 \\
\hline & & Kempnyia & $P$ & 45 & 148 & 16 & 5 \\
\hline & & Macrogynoplax & $P$ & 4 & 1 & 1 & \\
\hline \multirow[t]{9}{*}{ Hemiptera } & Helotrephidae & Neotrephes jackzewskii China, 1940 & $P$ & 60 & 4 & 25 & 1 \\
\hline & \multirow[t]{6}{*}{ Naucoridae } & Cryphocricos & $P$ & - & 14 & 21 & 2 \\
\hline & & $\begin{array}{l}\text { Limnocoris asper Nieser \& Lopez-Ruf, } \\
2001\end{array}$ & $P$ & 4 & - & - & 1 \\
\hline & & Limnocoris brasiliensis De Carlo, 1941 & $P$ & 9 & - & 3 & - \\
\hline & & $\begin{array}{l}\text { Limnocoris intermedius Nieser \& } \\
\text { Lopez-Ruf, } 2001\end{array}$ & $P$ & 8 & - & - & - \\
\hline & & Limnocoris pauper Montandon, 1897 & $P$ & 2 & 2 & 9 & - \\
\hline & & Limnocoris siolii De Carlo, 1966 & $P$ & - & 1 & - & 3 \\
\hline & Notonectidae & Enithares brasiliensis Spínola, 1836 & $P$ & 5 & - & 3 & - \\
\hline & Potamocoridae & Coleopterocoris hungerford De Carlo, 1968 & $P$ & - & - & 1 & - \\
\hline \multirow[t]{2}{*}{ Megaloptera } & \multirow[t]{2}{*}{ Corydalidae } & Corydalus sp.1 & $P$ & 1 & 14 & 12 & - \\
\hline & & Corydalus sp.2 & $P$ & 2 & 1 & 6 & - \\
\hline \multirow[t]{31}{*}{ Coleoptera } & Curculionidae & & SH & 2 & 1 & 1 & - \\
\hline & Dryopidae & & SH & 59 & 29 & 4 & - \\
\hline & \multirow[t]{4}{*}{ Dytiscidae } & Laccophilus ovatus Sharp, 1882 & $P$ & 1 & 1 & - & - \\
\hline & & cf. Laccornelus & $P$ & 22 & - & 8 & - \\
\hline & & Platynectes & $P$ & - & - & 1 & - \\
\hline & & Suphisellus & $P$ & - & 2 & - & - \\
\hline & \multirow[t]{25}{*}{ Elmidae } & Austrolimnius formosus (Sharp, 1882) & SC & - & 3 & 10 & 14 \\
\hline & & Austrolimnius laevigatus (Grouvelle, 1888) & SC & 2 & 6 & 23 & 11 \\
\hline & & Austrolimnius pilulus (Grouvelle, 1888) & SC & - & 4 & 3 & 1 \\
\hline & & aff. Cylloepus & CG & 4 & 3 & 1 & 1 \\
\hline & & Cylloepus & SC & 1 & 18 & 7 & 4 \\
\hline & & Gyrelmis & CG & 2 & 1 & 2 & 5 \\
\hline & & Heterelmis sp.1 & CG & 272 & 522 & 51 & 10 \\
\hline & & Heterelmis sp.2 & CG & 273 & 332 & 37 & 15 \\
\hline & & Heterelmis sp.3 & CG & 4 & 37 & 12 & \\
\hline & & Heterelmis sp.4 & SC & 1 & 316 & 6 & 23 \\
\hline & & Heterelmis sp.5 & SC & - & 93 & 6 & 3 \\
\hline & & Heterelmis sp.6 & SC & 1 & 17 & 2 & 2 \\
\hline & & Hexacylloepus & SC & 34 & - & 1 & 1 \\
\hline & & aff. Hexacylloepus & CG & 2 & 30 & 8 & 1 \\
\hline & & Hexanchorus sp.1 & CG & - & - & 1 & 4 \\
\hline & & Hexanchorus sp.2 & SC & - & 1 & - & 1 \\
\hline & & Macrelmis granosa (Grouvelle, 1896) & SC & - & 10 & 2 & 1 \\
\hline & & Macrelmis sp.1 & SC & - & 17 & 2 & 1 \\
\hline & & Macrelmis sp.2 & SC & 16 & 61 & 20 & 1 \\
\hline & & Macrelmis sp.3 & CG & 2 & 43 & 9 & 1 \\
\hline & & Microcylloepus sp.1 & CG & 1 & 7 & 4 & 16 \\
\hline & & Microcylloepus sp.2 & SC & 5 & 22 & 14 & 13 \\
\hline & & Microcylloepus sp.3 & CG & - & 2 & 1 & 10 \\
\hline & & Neoelmis sp.1 & CG & 17 & 18 & 114 & 6 \\
\hline & & Neoelmis sp.2 & SC & 8 & 34 & 34 & 4 \\
\hline
\end{tabular}

$\mathrm{LP}=$ litter from pool, $\mathrm{LR}=$ litter from riffle, $\mathrm{GR}=$ gravel and $\mathrm{RO}=$ rocks. $\mathrm{CG}=$ collector-gatherers, $\mathrm{CF}=$ collectorfilterers, $\mathrm{SC}=$ scrapers, $\mathrm{SH}=$ shredders, $\mathrm{P}=$ predators. 
Table 2. Continued...

\begin{tabular}{|c|c|c|c|c|c|c|c|}
\hline & & Taxa & FFG & $\mathrm{LP}$ & LR & GR & RO \\
\hline \multirow[t]{27}{*}{ Coleoptera } & \multirow[t]{13}{*}{ Elmidae } & Neoelmis sp.3 & CG & 1 & 5 & - & - \\
\hline & & aff. Neoelmis & $C G$ & 1 & 1 & 3 & - \\
\hline & & Phanocerus clavicornis Sharp, 1882 & SC & 27 & 256 & 10 & 7 \\
\hline & & Promoresia sp.1 & CG/SC & 7 & 24 & 4 & 28 \\
\hline & & Promoresia sp.2 & CG & 3 & 3 & 3 & 7 \\
\hline & & Stegoelmis & SC & - & - & 9 & - \\
\hline & & Xenelmis sp.1 & SC & - & 4 & 3 & 13 \\
\hline & & Xenelmis sp.2 & CG & 63 & 35 & 93 & 116 \\
\hline & & Xenelmis sp.3 & CG & 11 & - & 2 & 1 \\
\hline & & Elminae sp. 1 & CG & 5 & 3 & 125 & 14 \\
\hline & & Elminae sp. 2 & CG & - & 39 & 1 & - \\
\hline & & Elminae sp. 3 & CG & 30 & 4 & 5 & - \\
\hline & & Elminae sp. 4 & CG & - & 1 & 2 & - \\
\hline & Gyrinidae & Gyretes & $P$ & 21 & 1 & 3 & - \\
\hline & Hydraenidae & Hydraena & $P$ & 1 & 19 & 1 & 1 \\
\hline & \multirow[t]{6}{*}{ Hydrophilidae } & Berosus & $P$ & 1 & - & - & - \\
\hline & & Chasmogenus & $P$ & 6 & 1 & - & - \\
\hline & & Derallus & $P$ & 13 & - & - & - \\
\hline & & Enochrus & $P$ & - & 4 & - & - \\
\hline & & Oocyclus & $P$ & - & 1 & - & - \\
\hline & & Hydrophilinae sp. 1 & $P$ & - & 6 & - & - \\
\hline & Lutrochidae & Lutruchus & CG/SC & 20 & 19 & 1 & 1 \\
\hline & Psephenidae & & SC & 7 & 4 & 100 & 10 \\
\hline & Ptilidae & & - & 3 & 1 & - & 1 \\
\hline & Ptylodactilidae & & $\mathrm{SH}$ & 4 & - & 2 & - \\
\hline & Scirtidae & & CG & 1 & 14 & 26 & 2 \\
\hline & Staphilinidae & & $P$ & 4 & 24 & 8 & 1 \\
\hline \multirow[t]{25}{*}{ Trichoptera } & Anomalopsychidae & Contulma & SC & 4 & 20 & 8 & 8 \\
\hline & \multirow[t]{4}{*}{ Calamoceratidae } & Phylloicus sp. 1 & SH & 741 & 101 & 16 & 3 \\
\hline & & Phylloicus sp. 2 & SH & 59 & 107 & 2 & 8 \\
\hline & & Phylloicus sp. 3 & SH & 28 & 5 & 1 & - \\
\hline & & Phylloicus sp. 4 & SH & 8 & - & - & - \\
\hline & Ecnomidae & Austrotinodes & $\mathrm{CF}$ & 1 & - & - & - \\
\hline & Glossosomatidae & & SC & 18 & 14 & 120 & 40 \\
\hline & Hydrobiosidae & Atopsyche & $P$ & 4 & 163 & 33 & 27 \\
\hline & \multirow[t]{5}{*}{ Helicopsychidae } & Helicopsyche sp. 1 & SC & 23 & 18 & 137 & 27 \\
\hline & & Helicopsyche sp. 2 & SC & - & 5 & 14 & 24 \\
\hline & & Helicopsyche sp. 3 & SC & 5 & 7 & 61 & 6 \\
\hline & & Helicopsyche sp. 4 & SC & - & 7 & 153 & 62 \\
\hline & & Helicopsyche sp. 5 & SC & 1 & - & 3 & 6 \\
\hline & \multirow[t]{4}{*}{ Hydroptilidae } & Alisotrichia & $P$ & 1 & 41 & 28 & 224 \\
\hline & & Leucotrichia & SC & - & - & - & 4 \\
\hline & & Metrichia sp. 1 & CG/SC & 1 & 4 & - & 60 \\
\hline & & Metrichia sp. 2 & CG/SC & 6 & 9 & 4 & 129 \\
\hline & \multirow[t]{8}{*}{ Hydroptilidae } & Metrichia sp. 3 & CG/SC & - & 5 & 5 & 84 \\
\hline & & Metrichia sp. 4 & CG/R & 3 & 5 & 2 & 142 \\
\hline & & Metrichia sp. 5 & CG/R & - & - & - & 1 \\
\hline & & Neotrichia sp. 1 & $\mathrm{R}$ & 60 & 124 & 52 & 19 \\
\hline & & Neotrichia sp. 2 & $\mathrm{R}$ & 35 & 2 & & 1 \\
\hline & & Neotrichia sp. 3 & $\mathrm{R}$ & 40 & 37 & 35 & 3 \\
\hline & & Ochrotrichia (?) & CG & - & 1 & - & - \\
\hline & & Rhyacopsyche sp. 1 & CG/R & - & 1 & - & 13 \\
\hline
\end{tabular}

$\mathrm{LP}=$ litter from pool, $\mathrm{LR}=$ litter from riffle, $\mathrm{GR}=$ gravel and $\mathrm{RO}=$ rocks. $\mathrm{CG}=$ collector-gatherers, $\mathrm{CF}=$ collectorfilterers, $\mathrm{SC}=$ scrapers, $\mathrm{SH}=$ shredders, $\mathrm{P}=$ predators . 
Table 2. Continued...

\begin{tabular}{|c|c|c|c|c|c|c|c|}
\hline & & Taxa & FFG & LP & LR & GR & $\mathrm{RO}$ \\
\hline & & Rhyacopsyche sp. 2 & CG/R & - & - & - & 6 \\
\hline & Hydropsychidae & Blepharopus & CF & 2 & 31 & 11 & 6 \\
\hline & & Smicridea sp. 1 & CF & 51 & 1010 & 364 & 341 \\
\hline & & Smicridea sp. 2 & CF & - & 59 & 27 & 189 \\
\hline & & Smicridea sp. 3 & CF & - & 5 & 4 & 68 \\
\hline & & Smicridea sp. 4 & CF & - & 5 & 2 & 1 \\
\hline & & Leptonema sp. 1 & CF & - & 9 & 9 & 7 \\
\hline & & Leptonema sp. 2 & CF & - & - & 2 & 1 \\
\hline & & Leptonema sp. 3 & CF & - & - & 1 & 2 \\
\hline & & Macronema & CF & 31 & - & - & - \\
\hline & Leptoceridae & Atanatolica & $\mathrm{R}$ & 1 & 6 & 5 & 57 \\
\hline & & Grumichella & $\mathrm{R}$ & 38 & 68 & 39 & 1483 \\
\hline & & Nectopsyche sp.1 & CG & 525 & 996 & 20 & 14 \\
\hline & & Nectopsyche sp.2 & CG/SH & 29 & 11 & 4 & - \\
\hline & & Nectopsyche sp.3 & CG/SH & 8 & 5 & 2 & 2 \\
\hline & & Nectopsyche sp.4 & CG/SH & - & - & 2 & 1 \\
\hline & & Nectopsyche sp.5 & CG/SH & - & 8 & - & 1 \\
\hline & & Nectopsyche sp.6 & CG/SH & 49 & - & 1 & - \\
\hline & & Notalina & $\mathrm{SH}$ & 694 & 2 & 11 & - \\
\hline & & Oecetis sp.1 & $P$ & 105 & 3 & 12 & 3 \\
\hline & & Oecetis sp.2 & $P$ & 21 & 1 & 6 & - \\
\hline & & Triplectides & $\mathrm{SH}$ & 1839 & 42 & 73 & 4 \\
\hline & & Leptoceridae sp. 1 & CG & - & 1 & 1 & 2 \\
\hline & Odontoceridae & $\begin{array}{l}\text { Anastomoneura guahybae Huamantinco \& } \\
\text { Nessimian, } 2004\end{array}$ & $P$ & - & 2 & - & - \\
\hline & & Barypenthus concolor Burmeister, 1839 & $P$ & 16 & - & 24 & 1 \\
\hline & & Marilia sp.1 & $\mathrm{P} / \mathrm{R}$ & 44 & 8 & 17 & 2 \\
\hline & & Marilia sp.2 & $\mathrm{P} / \mathrm{R}$ & - & - & 10 & - \\
\hline & & Marilia sp.3 & $\mathrm{P} / \mathrm{R}$ & 5 & 1 & 6 & - \\
\hline & & Marilia sp.4 & $P / R$ & 1 & 5 & 2 & - \\
\hline & & Marilia sp.5 & $P / R$ & - & - & 1 & - \\
\hline & Philopotamidae & Chimarra & CF & - & 66 & 27 & 3 \\
\hline & & Wormaldia & CF & - & 21 & 12 & - \\
\hline & Polycentropodidae & Cyrnellus & CF & 2 & 10 & - & - \\
\hline & & Polycentropus & $\mathrm{CF} / \mathrm{P}$ & 26 & 2 & 6 & 1 \\
\hline & & Polyplectropus & CF & 2 & 8 & 16 & 1 \\
\hline & & Polycentropodidae sp. 1 & - & - & - & 1 & - \\
\hline & Sericostomatidae & Grumicha sp.1 & $\mathrm{R}$ & 106 & 554 & 44 & 28 \\
\hline & & Grumicha sp.2 & $\mathrm{R}$ & 76 & 796 & 133 & 24 \\
\hline & & Sericostomatidae sp. 1 & - & - & - & 4 & - \\
\hline & Xiphocentronidae & Xiphocentron & CF & 1 & 3 & 12 & 9 \\
\hline Lepidoptera & Pyralidae & & $\mathrm{SH}$ & 2 & 6 & 15 & 16 \\
\hline \multirow[t]{9}{*}{ Diptera } & Blephariceridae & & SC & - & - & 1 & 9 \\
\hline & Ceratopogonidae & & CG/P/SC & 592 & 527 & 58 & 49 \\
\hline & Chironomidae & & $\begin{array}{l}\text { CG/CF/ } \\
\text { SC/P }\end{array}$ & 7780 & 14462 & 4958 & 2757 \\
\hline & Culicidae & & CF & - & - & - & 1 \\
\hline & Dixidae & & CG & 12 & 47 & 25 & 16 \\
\hline & Dolichopodidae & & - & - & 2 & - & - \\
\hline & Empididae & & CG & 78 & 327 & 54 & 20 \\
\hline & Ephydridae & & CG & - & 2 & - & 1 \\
\hline & Psychodidae & & CG/SC & 9 & 277 & 26 & 248 \\
\hline
\end{tabular}

$\mathrm{LP}=$ litter from pool, $\mathrm{LR}=$ litter from riffle, $\mathrm{GR}=$ gravel and $\mathrm{RO}=$ rocks. $\mathrm{CG}=$ collector -gatherers, $\mathrm{CF}=$ collectorfilterers, $\mathrm{SC}=$ scrapers, $\mathrm{SH}=$ shredders, $\mathrm{P}=$ predators. 
Table 2. Continued...

\begin{tabular}{lllccccc}
\hline & Taxa & FFG & LP & LR & GR & RO \\
\hline Diptera & Simuliidae & & CF & 143 & 6837 & 199 & 6408 \\
& Stratiomyidae & & CG & 2 & 1 & - & - \\
& Tabanidae & & P & 1 & - & 4 & 1 \\
& Tipulidae & CG/SH/P & 110 & 103 & 388 & 16 \\
\hline
\end{tabular}

$\mathrm{LP}=$ litter from pool, $\mathrm{LR}=$ litter from riffle, $\mathrm{GR}=$ gravel and $\mathrm{RO}=$ rocks. $\mathrm{CG}=$ collector-gatherers, $\mathrm{CF}=$ collector filterers, $\mathrm{SC}=$ scrapers, $\mathrm{SH}=$ shredders, $\mathrm{P}=$ predators.
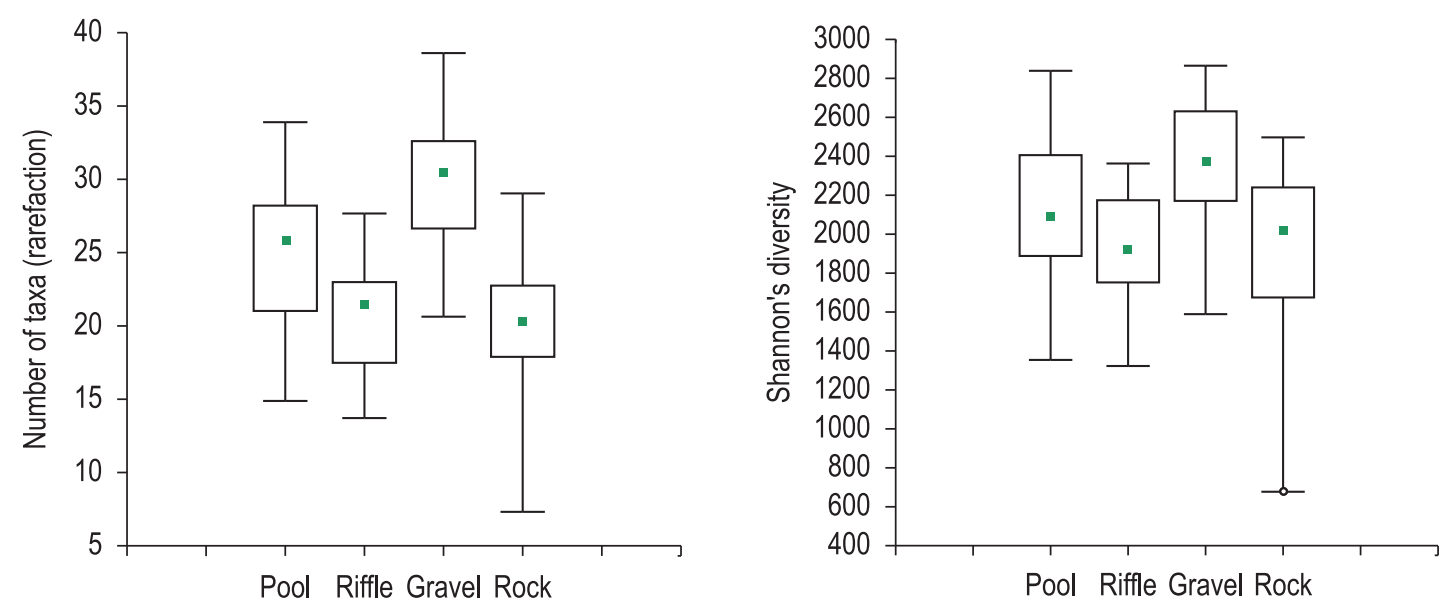

- Median $\square 25-75 \% \quad$ 工 Min-Max

Figure 2. Mean (Min-Max) rarefaction and Shannon's Diversity found to substrates studied in the streams at Serra da Bocaina.

taxa. In relation to groups of substrates, 27 taxa were characteristic of organic substrates, and 17 of inorganic substrates.

Cluster Analysis showed the aquatic insects are distributed mainly in relation to substrate type and food availability, forming characteristic habitat assemblages (Figure 3). Group A was formed by the inorganic substrates, mainly rocks in riffle areas, and group B formed by all remaining substrates. Group B was divided in two groups: B1 - formed by some pool litter and gravel samples, found mainly in pool areas, and B2 - formed by the majority litter samples independent of current velocity.

\subsection{Functional feeding groups}

Collector-gatherer was the main functional feeding group (40.64\%), followed by collectorfilterer $(26.04 \%)$, scraper $(18.20 \%)$, predator $(8.45 \%)$ and shredder $(6.67 \%)$ of the total abundance of individuals. The results of Indicator Value analysis $(\mathrm{p}<0.05)$ showed that collectorgatherers and shredders $(\mathrm{IV}=30.4$; IV $=68.6$, respectively) were indicatives for litter from pool; predator $(I V=31.5)$ was indicative for gravel substrates, and collector-filter and scrapers $(\mathrm{IV}=40.5$ and $\mathrm{IV}=34.4$, respectively $)$ were indicatives for rock substrates.

There were differences in abundance and proportion of the functional feeding groups among substrate types. Collector-gatherers $\left(\mathrm{F}_{3.68}=33.961\right.$, $\mathrm{P}<0.001)$ showed differences between litter from pool and riffles and rock and the other substrates (Tukey Test, $\mathrm{p}<0.05$; Figure 4a). Chironomidae, Elmidae larvae and some Ephemeroptera genera were the main representatives of this group. Collector-filterers $\left(\mathrm{F}_{3.68}=25.608 ; \mathrm{P}<0.001\right)$ showed differences between litter from pools and riffles, litter from riffles and gravel, and rock and other substrates (Tukey Test; $\mathrm{p}<0.05$; Figure $4 \mathrm{~b}$ ). Collector-filterer fauna was composed mainly by Simuliidae and some Tanytarsini (Chironomidae). Predators $\left(\mathrm{F}_{3.68}=7.2880 ; \mathrm{P}<0.001\right)$ showed differences between rock, litter from pool and gravel (Tukey Test, $\mathrm{p}<0.05$; Figure 4c). This group was poorly represented in the studied streams, reaching its highest representation in 
Table 3. Taxa indicators found to each substrate type and organic/inorganic susbtrates found at the Serra da Bocaina streams. $(\mathrm{p}<0.05)$.

\begin{tabular}{|c|c|c|c|}
\hline Pool Litter & Riffle Litter & Gravel & Rock \\
\hline Gyretes & Americabaetis & Argia & Alisotrichia \\
\hline Hetaerina & Anacroneuria & Austrolimnius laevigatus & Baetodes \\
\hline Hexacylloepus & Chimarra & Barypenthus & Grumichella \\
\hline Leptohyphodes & Chironomidae & Brechmorrhoga & Gripopteryx \\
\hline Limnetron debile & Contulma & Campylocia bocainensis & Metrichia sp.1 \\
\hline Limnetron sp.1 & Cyrnellus & Corydalus & Metrichia sp.2 \\
\hline Massartela alegrettae & Empididae & Cryphocricos & Metrichia sp.3 \\
\hline Massartela brieni & Grumicha sp.1 & Elminae sp.1 & Rhyacopsyche sp.1 \\
\hline Miroculis froehlich & Heterelmis sp.1 & Glossosomatidae & Smicridea sp.2 \\
\hline Neocordulia & Heterelmis sp.4 & Helicopsyche sp.1 & \\
\hline Neotrephes jackzewskii & Heterelmis sp.5 & Helicopsyche sp.4 & \\
\hline Notalina & Heterelmis sp. 6 & Limnocoris pauper & \\
\hline Oecetis sp.1 & aff. Hexacylloepus & Marilia sp.2 & \\
\hline Phylloicus sp.1 & Hydraena & Neoelmis & \\
\hline Phylloicus sp.3 & Hylister plaumanni & Polyplectropus & \\
\hline Polycentropus & Leptohyphes & Psephenidae & \\
\hline Triplectides & Lutruchus & Thraulodes itatiajanus & \\
\hline Ulmeritodes sp.1 & Macrelmis sp.1 & Thraulodes sp.1 & \\
\hline Xenelmis sp.2 & Macrelmis sp.2 & Tipulidae & \\
\hline \multirow[t]{6}{*}{ Zelusia principalis } & Macrelmis sp.3 & Traverhyhes & \\
\hline & Paragripopteryx & Tricorythodes & \\
\hline & Phanocerus & Tricorytopsis & \\
\hline & Phylloicus sp.2 & & \\
\hline & Smicridea sp.1 & & \\
\hline & Staphilinidae & & \\
\hline Organic substrates & & Inorganic substrates & \\
\hline Ceratopogonidae & Neocordulia & Austrolimius laevigatus & \\
\hline Chironomidae & Notalina & Austrolimius formosus & \\
\hline Cyrnellus & Phanocerus & Baetodes & \\
\hline Dryopidae & Phylloicus sp.1 & Elminae sp.1 & \\
\hline Empididae & Phylloicus sp.2 & Glossosomatidae & \\
\hline Gyretes & Phylloicus sp.3 & Grumichella & \\
\hline Hetaerina & Triplectides & Helicopsyche sp.2 & \\
\hline Heterelmis sp. 1 & Tupiperla & Helicopsyche sp.4 & \\
\hline Heterelmis sp. 2 & Ulmeritodes sp.1 & Metrichia sp.2 & \\
\hline Heterelmis sp. 5 & Zelusia principalis & Metrichia sp.3 & \\
\hline Limnetron debile & & Neoelmis & \\
\hline Limnetron sp. 1 & & Psephenidae & \\
\hline Lutruchus & & Rhyachopsyche sp.1 & \\
\hline Massartela alegrettae & & Thraulodes itatiajanus & \\
\hline Melanemerella brasiliana & & Thraulodes sp.1 & \\
\hline Miroculis froehlichi & & Tricorytopsis & \\
\hline Nectopsyche sp. 1 & & Xiphocentron & \\
\hline
\end{tabular}

gravel, where the main representatives were Hemiptera and Odonata. Scrapers $\left(\mathrm{F}_{3.68}=6.2899\right.$; $\mathrm{P}=0.000261)$ showed differences between rock and litter substrates (Tukey Test; $\mathrm{p}<0.05$; Figure $4 \mathrm{~d}$ ). The main representatives were Grumichella sp., Grumicha sp., Helicopsyche sp., Elmidae (adults) and some ephemeropterans.
Shredders $\left(\mathrm{F}_{3.68}=47.454 ; \mathrm{P}=0.000787\right)$ showed significant differences between litter from pools and the other substrates (Tukey Test; $\mathrm{p}<0$.05; Figure 4e). They were represented mainly by genera Notalina, Nectopsyche, Phylloicus, Triplectides, Melanemerella and Paragripopteryx. 


\begin{tabular}{|c|c|c|c|c|}
\hline \multicolumn{5}{|c|}{ Distance (Objective function) } \\
\hline 6 & 3923 & 7839 & 11756 & 15672 \\
\hline & & ion rems & & \\
\hline 10000 & 75000 & 50000 & 25000 & 0 \\
\hline
\end{tabular}

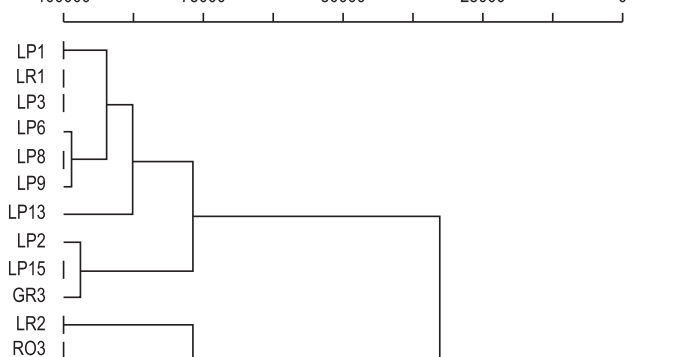

RO7

GR

GR8
LR12

LR14

LR9

RO15

LR3

LRT

LR8

LR15

LR16

LR17

LRT$$
\text { LR13 }
$$

GR9

LR10

LR18 |

GR1

LP17

R01

GR10

GR4

GR15

GR18

GR2

GR6

GR13

GR14

GR16

GR17

LP4

LP10

LP14
LP7

LP5

LP16

LP18

$\mathrm{RO} 2$

RO

R013

R011

R010

R014

R017

RO4

RO9

R016

$\mathrm{RO} 8$

R012

LP11

GR11

Figure 3. Cluster Analysis based on Bray-Curtis dissimilarity index using assemblages fauna abundance in the substrates collected at Serra da Bocaina streams. $\mathrm{LP}=$ litter from pool, $\mathrm{LR}=$ litter from riffles, $\mathrm{GR}=$ gravel, $\mathrm{RO}=$ rocks. Number 1 to 18 are the streams.

\section{Discussion}

\subsection{Spatial distribution}

Substrate type is a useful and convenient predictor of the abundance and diversity of benthic macroinvertebrates (Minshall, 1984; Beisel et al., 1998). Habitat structure may regulate species diversity at local scale, the more complex habitats being usually associated with greater richness values than simple ones (Downes et al., 1998). In the streams studied, most of the aquatic insect fauna was found associated to organic substrates, both in riffles and pools. This may be related to the capacity of these substrates to retain the most part of organic matter available inside a stream, providing shelter and abundant food. Leaf deposits are chosen preferentially in relation to mineral substrates by some taxa. According to Reice (1980) this choice is consistent with the detritivory of these groups and the high concentration of available resource in leaves. In this study, insect from riffle litters corresponded to $41.92 \%$ of the total sampled fauna. This abundance pattern in riffle mesohabitat, especially in litter, has been observed in several studies in streams from Atlantic Forest (Kikuchi and Uieda, 1998; Baptista et al., 2001a; CrisciBispo et al., 2007a; Buss et al., 2004; Silveira et al., 2006).

In riffle areas, litter is composed by large and relatively young leaves with little matter adhered, when compared with litter from pool areas. Insects that live in riffle areas use the current to get a continuous food flow and higher oxygenation, as in Smicridea and Chimarra, which built capture nets in fast flowing sites. More than $50 \%$ of the abundance of these genera is concentrated in such substrate. Although, Hylister plaumanni is reported by Da-Silva et al. (2010) as inhabitant in pool litter and sand in rithral stretches of rivers and Leptohyphes is reported by Francischetti et al. (2004) in litter from pools and gravel, these taxa were indicatives to riffle litter in the streams studied. As expected, Anacroneuria, Kempnyia and Paragripopteryx were indicative of riffle litter. Baptista et al (1998b) found that most Anacroneuria individuals preferred this substrate. Elmidae presented almost one half of the sampled individuals in riffle litter. Passos et al. (2003a), studying a small stream in Rio de Janeiro, reported this substrate as preferential to this family, especially to the genera Cylloepus, Heterelmis, Macrelmis, and Phanocerus.

Macroinvertebrate densities in streams are often correlated with the supply of coarse particulate 

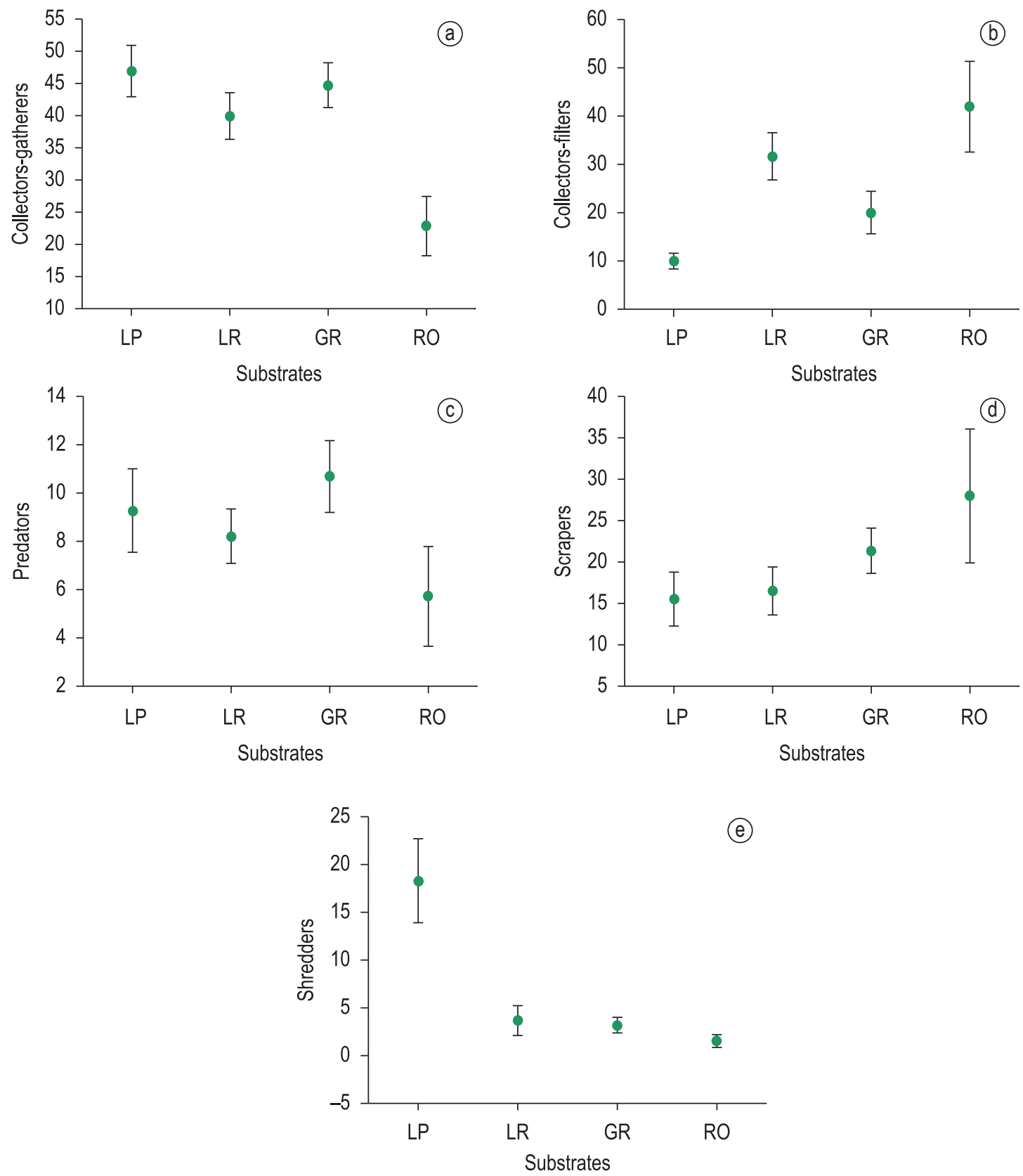

Figure 4. Mean (Max-Min) Tukey HSD test results found to each functional feeding groups and substrates $(\mathrm{p}<0.05)$. $\mathrm{LP}=$ pool litter, $\mathrm{LR}=$ riffle litter, $\mathrm{GR}=$ gravel and $\mathrm{RO}=$ rock .

organic material, mainly leaf from riparian vegetation (Townsend and Hildrew, 1988; Dudgeon and Wie, 1999). Litter from pools tends to be colonized by species with shredder habits such as Notalina, Oecetis, Phylloicus, and Triplectides. These genera use leaf pieces or small sticks from leaf packs both to feeding and to build their cases. Miroculis froehlichi and Leptohyphodes (Ephemeroptera) were abundant (90\% of individuals) in deposited litter. Askola, Massartela, Melanemerella, and Umeritoides were also indicators of this substrate. Several studies report these groups as dwellers of organic material in depositional areas of forested rivers (Molineri and Dominguez, 2003; Goulart and Callisto, 2005; Da-Silva et al., 2010). Hetaerina, Heteragrion, Limnetron, and Neocordulia (Odonata) are reported by Carvalho and Nessimian (1998) as inhabitants of both erosional and depositional areas with presence of detritus, roots and macrophytes. In Serra da Bocaina streams, these taxa occurred mainly in pool litter.

Gravel substrate had the highest taxonomic richness and 22 characteristic taxa. This substrate can represent a transition between sand and 
rocky substrates. In our study, gravel samples were composed by a mix of small cobbles and pebbles collected in fast and moderate current. This variability of particle size and current velocity coupled with low stability of the sediment may have been responsible for high species richness and low density of individuals found in gravel substrates. Among the Ephemeroptera indicators of gravel, Thraulodes itatiajanus lives in rocky mid-size streams with strong current, taking shelter among pebbles in the streambed (Da-Silva, 2003), whereas Traverhyphes and Tricorytopsis are found in areas with strong current between rocks and litter riffle (Baptista et al., 2006). In this study the Thraulodes taxa had highest density in gravel. Although few, most individuals of Brechmorrhoga were sampled in gravel substrate, confirming their preference for hard substrates in riffle areas as observed by Assis et al. (2004) in Ubatiba River, Maricá, Rio de Janeiro State. Corroborating Passos et al. (2003b), Neoelmis larvae and adults occurred in litter from riffles and stones.

Several studies found that rocky substrates supported higher density and biomass of macroinvertebrates than sand and leaf packs (e.g. Hynes, 1970; Brown and Brussock, 1991; Velásquez and Miserendino, 2003). In the streams studied here, lower richness and abundance were found in rocky substrate. Rocky substrate was preferential for scrapers and collectors-filterers. Baetodes, Grypopteryx, Hydroptilidae (Alisotrichia, Metrichia and Rhyachopsyche sp.1), Smicridea sp.2 and Grumichella were the major representatives of this substrate.

Aquatic insect distribution was influenced mainly by substrate type. Independently of river or altitude studied, substrates of same type have similar faunal assemblages, suggesting that availability and nature of substrate are the main factors which govern the benthic macroinvertebrate colonization (Hynes, 1970; Minshall, 1984). Beisel et al. (1998) found that nature of substrate also appeared to exert a strong influence on community structure. Some studies in Brazil have also shown that the distribution of macroinvertebrates was influenced primarily by substrate type rather than environmental integrity, sampling period or river order (Buss et al., 2004; Baptista et al., 1998a; Costa and Melo, 2008). The substrates are colonized by individuals that show similar morphological and functional characteristics that allow than to live in these habitats. Many species can colonize more than one substrate, but in general the substrates show distinct assemblages.
The results found in this study corroborate those of Minshall and Petersen (1985) and Buss et al. (2004), which showed that macroinvertebrates assemblages are not random assemblages of species.

\subsection{Functional feeding groups}

As expected, the functional feeding group distribution showed variation across habitats. Collector-gatherers displayed higher relative proportion in litter from pool and gravel. Collectorsgathers is one poorly understood functional feeding groups, mainly because little is known about the sources of FPOM (Cummins, 1974). This functional feeding group was predominant in all rivers, mainly in litter from pools. This result is similar to those of Baptista et al. (1998b) and Silveira et al. (2006). Collectors-filters have filtration mechanisms and feed on fine particulate organic matter (FPOM) or dissolved organic matter (DOM) present in suspension in water column. This trophic group was the second most abundant, being more concentrated in rock substrate and litter from riffle.

According Cummins (1973), scrapers cut or grazes the periphyton (biofilm) and fine organic matter deposited or attached to stones and vegetation. The biofilm is composed not only by algae, but also by bacteria, fungi and organic matter embedded in a mucilaginous matrix (Graça, 2001). Scrapers constituted the third most important functional feeding group in this study. They showed higher relative proportion in rock substrates in riffle area, where there are appropriate conditions to biofilm growth.

Predators showed low participation in the studied streams. In general, this guild is well represented in lotic and lentic environments, being composed mainly by Coleoptera, Hemiptera, Odonata, Plecoptera and some crustaceans. CrisciBispo et al. (2007a) studying the EPT functional feeding structure in two mesohabitats (pool and riffles litter), found predators represented $23.12 \%$ in riffle litter. Although well represented in the neotropic region, predators guild often have few individuals in the streams of Southeastern Brazil. Silveira et al. (2006) found the highest predator abundance in fourth order stream reach with $13.89 \%$, and Crisci-Bispo et al. (2007b) studying EPT fauna found predators compound only 8.72 and $2.21 \%$ respectively in two streams.

Baptista et al. (1998b), studied the River Continuum Concept in Macaé river basin (Rio de Janeiro State) and found that shredders 
represented between 19 and $35.51 \%$ in streams of first to fourth order. Shredders and collectors were the major primary consumers in forest streams, providing the main link between the organic inputs and the predatory invertebrates and vertebrates (Cheshire et al., 2005). In this study, shredders had the lower relative participation (6.67\%). Other studies in Southeastern Brazil revealed the occurrence of few shredders groups in rivers (e.g. Moulton and Magalhães, 2003; Gonçalves et al., 2006; Silveira et al., 2006). Some studies (e.g. Dobson et al., 2002; Wantzen and Wagner, 2006) had demonstrated that shredders are scarce in tropical regions. Cheshire et al. (2005) pointed out that most of the common shredder taxa from temperate systems are lacking in the tropics streams, like some stoneflies (Taeniopterygidae, Nemouridae, Leuctridae and Capniidae) and caddisflies (Limnephilidae and Sericostomatidae). Although, Limnephilidae and Sericostomatidae have representatives in southeastern region of Brazil, the species have different habits in relation to their cofamilial species in temperate region. Irons et al. (1994) suggested that shredding may be less important in tropical systems because there are alternative decomposition pathways for leaves, such as faster microbial processing due to higher temperatures. Some studies reported that in tropics leaves are thought to be a more recalcitrant food source for shredding organisms because leaves of tropical trees show high concentration of toxic compounds (e.g. Boyero et al., 2009; Wantzen et al., 2002).

\section{Conclusion}

The substrate type is one of the main environmental factors that affects distribution and abundance of aquatic insects. Our results corroborated this affirmation, showing that independently of stream size or altitude, similar substrates have similar assemblages. The abundance and relative proportion of the functional feeding group showed variation across habitats. In general, collector-gathers and shredders were predominant in pool substrates, collector-filters in riffle substrate and scrapers in hard substrates. However, in this study the mouthparts and gut contents of specimens were not examined, and thus inferences regarding the contribution of FFGs may be inaccurate. Being so, more detailed investigations about functional feeding categorization in southeastern Brazil need to be developed. The variation in functional feeding group distribution across habitats may have significant implications in understanding the spatial changes in stream structure communities.

\section{Acknowledgements}

The authors thank The Nature Conservancy of Brazil and Conselho Nacional de Desenvolvimento Científico e Tecnológico (CNPq-CT-HIDRO) for financial support given to the first author, and $\mathrm{CNPq}$ for fellowship to the second author. Instituto Chico Mendes de Biodiversidade (ICMBIO-MMA) and Serra da Bocaina National Park for collect permits. The authors are also grateful to Allan Paulo Moreira dos Santos and Felipe Ferraz Figuereido Moreira for English corrections.

\section{References}

ANGRISANO, EB. 1995. Insecta Trichoptera. In: LOPRETTO, EC. and TELL, G., eds. Ecosistemas de Aguas Continentales: Metodologia para su Estudio. La Plata: Ediciones Sur. vol. 3, p.1199-1224.

ASSIS, JCF., CARVALHO, AL. and NESSIMIAN, JL. 2004. Composiçáo e preferência por microhábitat de imaturos de Odonata (Insecta) em um trecho de baixada do Rio Ubatiba, Maricá - RJ. Revista Brasileira de Entomologia, vol. 48, no. 2, p. 273-282.

BAPTISTA, DF., BUSS, DF., DORVILLÉ, LFM. and NESSIMIAN, JL. 1998a.O conceito de continuidade de rios é válido para rios de Mata Attântica no sudeste do Brasil?. In NESSIMIAN, JL. and CARVALHO, Al., eds. Ecologia de Insetos Aquáticos, Series Oecologia Brasiliensis. Rio de Janeiro. vol. 5: Insetos Aquáticos, p. 209-222.

BAPTISTA, DF., DORVILLÉ, LFM., BUSS, DF., NESSIMIAN, JL. and SOARES, LHJ. 1998b. Distribuição de comunidade de insetos aquáticos no gradiente longitudinal de uma bacia fluvial do sudeste brasileiro. In NESSIMIAN, JL. and CARVALHO, Al., eds. Ecologia de Insetos Aquáticos, Series Oecologia Brasiliensis. Rio de Janeiro. vol. 5: Insetos Aquáticos, p. 191-207.

BAPTISTA, DF., BUSS, DF., DORVILLÉ, LFM. and NESSIMIAN, JL. 2001a. Diversity and habitat preference of aquatic insects along the longitudinal gradient of the Macaé river basin, Rio de Janeiro, Brazil. Revista Brasileira de Biologia, vol. 61, no. 2, p. 249-258.

BAPTISTA, DF., DORVILLÉ, LFM., BUSS, DF. and NESSIMIAN, JL. 2001b.Spatial and temporal organization of aquatic insects assemblages in the longitudinal gradient of a tropical river. Revista Brasileira de Biologia, vol. 61, no. 2, p. 295-304. http:// dx.doi.org/10.1590/S0034-71082001000200012

BAPTISTA, DF., BUSS, DF., DIAS, LG., NESSIMIAN, JL., DA-SILVA, ER., DE MORAES NETO, AHA., DE CARVALHO, SN., DE OLIVEIRA, MA. and RADE, LR. 2006. Functional feeding groups of 
Brazilian Ephemeroptera nymphs: ultrastructure of mouthparts. Annales de Limnologie-International Journal of Limnology, vol. 42, no. 2, p. 87-96.

BAPTISTA, DF., BUSS, DF., EGLER, M., GIOVANELLI, A., SILVEIRA, MP. and NESSIMIAN, JL. 2007. A multimetric index based on benthic macroinvertebrates for evaluation of Atlantic Forest streams at Rio de Janeiro State, Brazil. Hydrobiologia, vol. 575, p. 83-94.

BELLE, J. 1992. Studies on ultimate instar larvae of Neotropical Gomphidae, with the description of Tibiagomphus gen. nov. (Anisoptera). Odonatologica, vol. 21, no. 3, p. 1-24.

BEISEL, JN., USSEGLIO-POLATERA, P., THOMAS, S. and MORETEAU, JS. 1998. Stream community structure in relation to spatial variation: the influence of microhabitat characteristics. Hydrobiologia, vol. 389, p. 73-88. http://dx.doi. org/10.1023/A:1003519429979

BENFIELD, EF. 1997. Comparison of litterfall input to streams. In WEBSTER, JR. and MEYER, JL., eds. Stream organic matter budgets. Journal of North American Benthological Society, vol. 16, no. 1, p. 104-108.

BOYERO, L., RAMÍREZ, A., DUDGEON, D. and PEARSON, DG. 2009. Are tropical streams really different? Journal of North American Benthological Society, vol. 28, no. 2, p. 397-403. http://dx.doi. org/10.1899/08-146.1

BROWN, AV. and BRUSSOCK, PP. 1991. Comparison of benthic cinvertebrates between riffles and pools. Hydrobiologia, vol. 220, p. 99-108. http://dx.doi. org/10.1007/BF00006542

BUSS, DF., BAPTISTA, DF., NESSIMIAN, JL. and EGLER, M. 2004. Substrate specificity, environmental degradation and disturbance structuring macroinvertebrate assemblages in neotropical streams. Hydrobiologia, vol. 518, p. 179-188. http://dx.doi.org/10.1023/ B:HYDR.0000025067.66126.1c

CALLISTO, M., MORENO, P. and BARBOSA, FAR. 2001. Habitat diversity and benthic functional trophic groups at Serra do Cipó, Southeast Brazil. Revista Brasileira de Biologia, vol. 61, no. 2, p. 259-266. http://dx.doi.org/10.1590/S003471082001000200008

CARVALHO, AL. and CALIL, ER. 2000. Chaves de identificação para as famílias de Odonata (Insecta) ocorrentes no Brasil, adultos e larvas. Papéis Avulsos de Zoologia, vol. 41, no. 15, p. 223-241.

CARVALHO, AL. and NESSIMIAN, JL. 1998. Odonata do Estado do Rio de Janeiro, Brasil: hábitats e hábitos das larvas In NESSIMIAN, JL. and CARVALHO, Al., eds. Ecologia de Insetos Aquáticos, Series Oecologia Brasiliensis. Rio de Janeiro. vol. 5: Insetos Aquáticos, p. 03-28.
CARVALHO, AL. 1989. Description of the larvae of Neuraeschna costalis (Burmeister), with notes on its biology, and a key to the genera of Brazilian Aeshnidae larvae (Anisoptera). Odonatologica, vol. 18 , no. 4, p. 325-332.

CARVALHO, AL., WERNECK-DE-CARVALHO, P. and CALIL, ER. 2002. Description of the larvae of two species of Dasythemis Karsch, with a key to the genera of Libellulidae occurring in the states of Rio de Janeiro and São Paulo, Brazil (Anisoptera). Odonatologica, vol. 31, no. 1, p. 23-33.

CHESHIRE, K., BOYERO, L. and PEARSON, RG. 2005. Food webs in tropical Australia streams: shredders are not scarce. Freshwater Biology, vol. 50, p. 748-769. http://dx.doi.org/10.1111/j.13652427.2005.01355.x

COSTA, SS and MELO, AS. 2008. Beta diversity in stream macroinvertebrate assemblages: among-site and among-microhabitat components. Hydrobiologia, vol. 598, p. 131-138.

CRISCI-BISPO, VLC, BISPO, PC. and FROEHLICH, CG. 2007a. Ephemeroptera, Plecoptera and Trichoptera assemblages in litter in a mountain stream of the Atlantifc Rainforest from southeastern Brazil. Revista Brasileira de Zoologia, vol. 24, no. 3, p. 545-551. http://dx.doi.org/10.1590/S010181752007000300004

CRISCI-BISPO, VLC, BISPO, PC. and FROEHLICH, CG. 2007b. Ephemeroptera, Plecoptera and Trichoptera assemblages in two Atlantifc Rainforest streams, Southeastern Brazil. Revista Brasileira de Zoologia, vol. 24, no. 2, p. 312-318-551.

CUMMINS, KW. and KLUG, MJ. 1979. Feeding ecology of stream invertebrates. Annual Review of Ecology and Systematics, vol. 10, p. 147-172. http:// dx.doi.org/10.1146/annurev.es.10.110179.001051

CUMMINS, KW. and LAUF, GH. 1969. The influence of substrate particle size on the microdistribution of stream macrobenthos. Hydrobiologia, vol. 34, p. 145-181. http://dx.doi.org/10.1007/BF00141925

CUMMINS, KW. 1973. Trophic relations of aquatic insects. Annual Review of Entomology, vol. 18, p. 183-206. http://dx.doi.org/10.1146/annurev. en.18.010173.001151

CUMMINS, KW. 1974. Structure and function of stream ecosystems. BioScience, vol. 24, p. 631-641. http://dx.doi.org/10.2307/1296676

DA-SILVA, ER. 2003. Ninfas de Thraulodes Ulmer, 1920 (Insecta: Ephemeroptera: Leptophlebiidae) ocorrentes no Estado do Rio de Janeiro, Brasil. Biota Neotropica, vol. 3, no. 2. Available from: <http:// www.biotaneotropica.org.br/v3n2/pt/abstract?articl e+BN01803022003>.

DA-SILVA, ER., SALLES, FF., NESSIMIAN, JL. and COELHO, LBN. 2003. A identificação das famílias de Ephemeroptera (Insecta) ocorrentes no Estado do Rio de Janeiro: Chave pictórica para as ninfas. 
Boletim do Museu Nacional, Nova Série., Zoologia., vol. 508, p. 1-6.

DA-SILVA, ER., NESSIMIAN, JL. and COELHO, LBN. 2010. Leptophlebiidae ocorrentes no Estado do Rio de Janeiro, Brasil: hábitats, meso-hábitats e hábitos das ninfas (Insecta: Ephemeroptera). Biota Neotropica, vol. 10, no. 4, p. 87-93.

DE SOUZA, ML. and MOULTON, TP. 2005. The effects of shrimps on benthic material in a brazilian island stream. Freshwater Biology, vol. 50, p. 592-602. http://dx.doi.org/10.1111/j.13652427.2005.01348.x

DIAS, LG., SALLES, FF., FRANCISCHETTI, CN. and FERREIRA, PSF. 2006. Key to the genera of Ephemerelloidea (Insecta: Ephemeroptera) from Brazil. Biota Neotropica, vol. 6, no. 1. Available from: <http://www.biotaneotropica.org.br/v6n1/ pt/abstract?Identification-key+bn00806012006. issn 1676-0611>.

DOBSON M., MATHOOKO, JM., MAGANA, A. and NDEGWA, FK. 2002. Macroinvertebrate assemblages and detritus processing in Kenyan highland streams: more evidence for the paucity of shredders in the tropics? Freshwater Biology, vol. 47, p. 909-919. http://dx.doi.org/10.1046/j.13652427.2002.00818.x

DOWNES, BJ., LAKE, PS., SCHREIBER, ESG. and GLAISTER, A. 1998. Habitat structure and regulation of local species diversity in a stony, upland stream. Ecological Monographs, vol. 68, no. 2, p. 237-257. http://dx.doi.org/10.1890/00129615(1998)068[0237:HSAROL]2.0.CO;2

DUDGEON, D. and WIE, KKY. 1999. Leaf litter in a tropical stream: food or substrate for macroinvertebrates? Archiv für Hydrobiologie, vol. 146 , no. 1, p. 65-82.

DUFRÊNE, M. and LEGENDRE, P. 1997. Species assemblages and indicator species: The need for a flexible asymmetrical approach. Ecological Monographs, vol. 67, no. 3. p. 345-366.

ELLIOT, JM. 1977. Some methods for statistical analysis of samples of benthic invertebrates. 2th ed. London: Freshwater Biological Association. 160 p. (Scientific Publication no. 25).

FRANCISCHETTI, CN., Da-SILVA, ER., SALLES, FF. and NESSIMIAN, JL. 2004. A efemeropterofauna (Insecta: Ephemeroptera) do trecho ritral inferior do Rio Campo Belo, Itatiaia, RJ: composiçáo e mesodistribuição. Lundiana, vol. 5, no. 1, p. 33-39.

GONÇALVES, JF., GRAÇA, MAS. and CALLISTO, M., 2006. Leaf-litter breakdown in 3 streams in temperate, mediterranean, and tropical Cerrado climates. Journal of North American Benthological Society, vol. 25, no. 2, p. 344-355. http://dx.doi. org/10.1899/0887-3593(2006)25[344:LBISIT]2. $0 . \mathrm{CO} ; 2$
GOULART, M. and CALLISTO, M. 2005. Mayfly distribution along a longitudinal gradient in Serra do Cipó, southeastern Brazil. Acta Limnologica Brasiliensia, vol. 17, no. 1, p. 1-13.

GRAÇA, MAS. 2001. The role of invertebrates on leaf litter decomposition in streams - a review. Internaternational Review of Hydrobiology, vol. 86, no. 4-5, p. 383-393. http://dx.doi.org/10.1002/15222632(200107)86:4/5\%3C383::AIDIROH383\%3E3.0.CO;2-D

GRAÇA, MAS., CRESSA, C., GESSNER, MO., FEIO, MJ., CALLIES, KA. and BARRIOS, C. 2001. Food quality, feeding preferences, survival and growth of shredders from temperate and tropical streams. Freshwater Biology, vol. 46, p. 947-957. http://dx.doi. org/10.1046/j.1365-2427.2001.00729.x

HYNES, HBN. 1970. The ecology of running waters. University of Toronto Press. 555 p.

IRONS, JG., OSWOOD, MW., STOUT, RJ. and PRINGLE, CM. 1994. Latitudinal patterns in leaf litter breakdown: is temperature really important? Freshwater Biology, vol. 32, p. 401-411. http://dx.doi. org/10.1111/j.1365-2427.1994.tb01135.x

KIKUCHI, RM. and UIEDA, VS. 1998. Composição da comunidade de invertebrados de um ambiente lótico tropical e sua variação espacial e temporal. In NESSIMIAN, JL. and CARVALHO, Al., eds. Ecologia de Insetos Aquáticos, Series Oecologia Brasiliensis. Rio de Janeiro. vol. 5: Insetos Aquáticos, p. 157-173.

LUDWIG, JA. and REYNOLDS, JF. 1988. Statistical Ecology: a primer on methods and computing. John Wiley and Sons, Inc. 337 p.

MCCUNE, B. and MEFFORD, MJ. 1999. Multivariate analysis of Ecological Data. Version 4.14. Gleneden Beach: MJM Software. 1999.

MERRITT, RW. and CUMMINS, KW. 1996. An introduction to the Aquatic Insects of North America. 3th ed. Kendall: Hunt Publishing Company. 862 p.

MERRITT, RW., CUMMINS, KW. and ANDRADE, PCN. 2005. The use of invertebrate functional groups to characterize ecosystem attributes in selected streams and rivers in southeast Brazil. Studies on Neotropical Fauna and Environmental, vol. 40, no.1, p. 71-90.

MINSHALL, GW. 1984. Aquatic insect- substratum relationships. In RESH, VH. and ROSENBERG, DM., eds. The ecology of Aquatic Insects. New York: Praeger Publishers. p: 358-400.

MINSHALL, GW. And PETERSEN, RC. 1985. Towards a theory of macroinvertebrate community structure in stream ecosystems. Archiv für Hydrobiology, vol. 104, p.49-76.

MOLINERI, C. and DOMÍNGUEZ, E. 2003. Nymph and egg of Melanemerella brasiliana (Ephemeroptera: Ephemerelloidea: Melanemerellidae), with comments 
on its systematic position and the higher classification of Ephemerelloidea. Journal of North American Benthological Society, vol. 22, no. 2, p. 263-275. http://dx.doi.org/10.2307/1467997

MOULTON, TP. and MAGALHÃES, SAP. 2003. Responses of leaf processing to impacts in streams in Atlantic Rain Forest, Rio de Janeiro, Brazil - a test of the Biodiversity-ecosystem Functioning relationship? Brazilian Journal of Biology, vol. 63, no. 1, p. 87-95. http://dx.doi.org/10.1590/S151969842003000100012

NIESER, N. and MELO, AL. 1997. Os Heterópteros Aquáticos de Minas Gerais: Guia Introdutório com Chave de Identificação para as Espécies de Nepomorpha e Gerromorpha. Belo Horizonte: Ed. UFMG. 180 p.

OLIFIERS, MH., DORVILLÉ, LFM., NESSIMIAN, JL. and HAMADA, N. 2004. A key to Brazilian genera of Plecoptera (Insecta) based on nymphs. Zootaxa, vol. 651, p. 1-15.

PARDO, I. and ARMITAGE, PD. 1997. Species assemblages as descriptors of mesohabitats. Hydrobiologia, vol. 344, p. 111-128. http://dx.doi. org/10.1023/A:1002958412237

PASSOS, MIS., NESSIMIAN, JL. and DORVILLÉ, LFM. 2003a. Distribuição espaço-temporal da comunidade de Elmidae (Coleoptera) em um rio na Floresta da Tijuca, Rio de Janeiro, RJ. Boletin do Museu Nacional, Nova Série, Zoologia, vol. 509, p. 1-9.

PASSOS, MIS., NESSIMIAN, JL. and DORVILLÉ, LFM. 2003b. Life strategies in an elmid (Insecta: Coleoptera: Elmidae) community from a first order stream in the Atlantic Forest, southeastern Brazil. Acta Limnologica Brasiliensia, vol. 15, no. 2, p. 29-36.

PES, AMO. HAMADA, N. and NESSIMIAN, JL. 2005. Chaves de identificação de larvas para famílias e gêneros de Trichoptera (Insecta) da Amazônia Central, Brasil. Revista Brasileira de Entomologia, vol. 49, no. 2, p. 181-204.

REICE, SR. 1980. The role of substratum in benthic macroinvertebrate microdistribution and litter decomposition in a woodland stream. Ecology, vol. 61, no. 3, p. 580-590. http://dx.doi. org/10.2307/1937424

ROHLF, FJ. 1992. NTSYS-pc Numerical taxonomy and Multivariate Analysis System. New York: University of New York, Setauret. 244 p.

ROQUE, FO. and TRIVINHO-STRIXINO, S. 2001. Benthic macroinvertebrates in mesohabitats in different spatial dimensions in a first order stream (São Carlos- SP). Acta Limnolica Brasiliensia, vol. 13, no. 2, p. 69-77.

SALLES, FF., DA-SILVA, ER., SERRÃO, JE. and FRANCISCHETTI, CN. 2004. Baetidae (Ephemerotera) na Região Sudeste do Brasil: Novos registros e chave para os gêneros no estágio ninfal. Neotropical Entomolgy, vol. 33, no. 5, p. 725-735.
SILVEIRA, MP., BAPTISTA, DF., BUSS, DF., NESSIMIAN, JL. and EGLER, M. 2005. Application of Biological measures for stream integrity assessment in South-East Brazil. Environmental Monitoring and Assessment, vol. 101, p. 117-128.

SILVEIRA, MP., BUSS, DF., NESSIMIAN, JL. and BAPTISTA, DF. 2006. Spatial and temporal distribution of benthic macroinvertebrates in a southeastern Brazilian river. Brazilian Journal of Biology, vol. 66, no. 2B, p. 623-632. http://dx.doi. org/10.1590/S1519-69842006000400006

STATSOFT INC. 2001. Statistica (data analysis software system). Version 6. Available from: <www.statsoft. com>.

SUBRAMANIAN, KA. and SIVARAMAKRISHNAN, KG. 2005. Habitat and microhabitat distribution of stream insect communities of the Western Ghats. Current Science, vol. 89, no. 6, p. 976-987.

TOWNSEND, CR. and HILDREW, AG. 1988. Pattern and process in low order acid streams. Verhandlungen des Internationalen Verein Limnologie, vol. 23, p. 1267-1271.

VANNOTE, RL., MINSHALL, GW., CUMMINS, KW., SEDELL, JR. and CUSHING, CE. 1980. The river continuum concept. Canadian Journal of Fisheries and Aquatic Science, vol. 37, p. 130-137. http://dx.doi.org/10.1139/f80-017

VELÁSQUEZ, SM. and MISERENDINO, ML. 2003. Habitat type and macroinvertebrate assemblage in low order Patagonian streams. Archiv für Hydrobiologie, vol. 158, no. 4, p. 461-483. http:// dx.doi.org/10.1127/0003-9136/2003/0158-0461

WALLACE, JB. and WEBSTER, JR. 1996. The role of macroinvertebrate in stream ecosystem function. Annual Review of Entomology, vol. 41, p. 115-1139. http://dx.doi.org/10.1146/annurev. en.41.010196.000555

WANTZEN, KM., WAGNER, R., SUETFELD, R. and JUNK, WJ. 2002. How do plant-herbivore interactions of trees influence coarse detritus processing by shredders in aquatic ecosystems of different latitudes? Verhandlungen des Internationalen Verein Limnologie, vol. 28, p. 1-7.

WANTZEN, KM. and WAGNER, R. 2006. Detritus processing by invertebrate shredders: a neotropicaltemperate comparison. Journal of North American Benthologial Society, vol. 25, no. 1, p. 216-232. http:// dx.doi.org/10.1899/0887-3593(2006)25[216:DPBI $\mathrm{SA}] 2.0 . \mathrm{CO} ; 2$

WARD, JV. 1992. Aquatic insects ecology 1: Biology and habitat. Singapore: John Wiley and Sons, Inc. 438 p.

WILLIAMS, DD. and FELTMATE, BW. 1994. Aquatic Insects. Wallingford: CAB International. 358 p.

Received: 12 July 2010 Accepted: 11 June 2011 